\section{Predicting outcomes in interstitial lung disease}

\section{S1 ELEVATED NOCTURNAL OXYGEN DESATURATION INDEX PREDICTS HIGHER SHORT-TERM MORTALITY IN INTERSTITIAL LUNG DISEASE}

TJ Corte, S Talbot, SJ Wort, AU Wells. Royal Brompton Hospital, London, UK

doi:10.1136/thx.2009.127050a

Nocturnal desaturation is present in $30-40 \%$ of patients with interstitial lung disease (ILD), and may be associated with poorer quality of life. However, the prognostic impact of nocturnal desaturation is not well established in patients with ILD.

Aim To assess the prognostic utility of parameters of nocturnal desaturation across ILD.

Methods Patients with ILD with overnight oximetry from June 2006 to August 2008 were reviewed ( $\mathrm{n}=139$, age $59 \pm 13$ years, 51 male, body mass index (BMI) $28 \pm 7 \mathrm{~kg} / \mathrm{m}^{2}$ ). All patients had corresponding pulmonary function, 133 had echocardiography and 123 had brain natriuretic peptide (BNP). Patients were followed to death, last follow-up or 19 March 2009. Outcome was evaluated for mortality using Cox proportional hazards analysis. The desaturation index (DI) was defined as the number of desaturation events $>4 \%$ per hour of study time. Pulmonary hypertension $(\mathrm{PH})$ was defined as systolic pulmonary arterial pressure $\geqslant 50 \mathrm{~mm} \mathrm{Hg}$ or right heart dilatation on echocardiography.

Results Nocturnal desaturation was present in 50 (36\%) patients, and mean minimum $\mathrm{SpO}_{2}$ was $76.3 \pm 9.9 \%$. Twenty-eight $(20 \%)$ had $\mathrm{PH}$ on echocardiography. An increased DI was associated with higher mortality independent of age, gender and BMI (hazards ratio (HR) $1.04 ; 95 \%$ CI 1.00 to $1.08 ; p=0.007)$. In separate models, DI and (1) elevated BNP (HR 1.01; CI 1.00 to 1.02; p =0.03); (2) PH on echocardiography (HR 23.27; CI 1.28 to 8.31; $\mathrm{p}=0.01$ ); and (3) daytime resting $\mathrm{SpO}_{2}$ ( $\mathrm{HR}$ 0.92; CI 0.85 to $0.99 ; \mathrm{p}=0.03$ ) independently predicted mortality following adjustment for age, gender and BMI.

Conclusion Elevated nocturnal DI predicts higher mortality across patients with ILD, independent of BNP and $\mathrm{PH}$ on echocardiography and resting daytime $\mathrm{SpO}_{2}$. DI may be an important prognostic consideration for patients with ILD, and warrants further prospective study.

\section{S2 GENE SET ANALYSIS OF LUNG SAMPLES FROM PROGRESSIVE, FIBROTIC PULMONARY SARCOIDOSIS SHOWS A PATTERN SIMILAR TO HYPERSENSITIVITY PNEUMONITIS}

${ }^{1} \mathrm{~S}$ Sanderson, ${ }^{2} \mathrm{HE}$ Lockstone, ${ }^{1} \mathrm{~N}$ Kulakova, ${ }^{2} \mathrm{D}$ Baban, ${ }^{3} \mathrm{Cl}$ Newbold, ${ }^{1} \mathrm{AJ}$ McMichael, ${ }^{4} \mathrm{~L}-\mathrm{P}$ Ho. ${ }^{1} \mathrm{MRC}$ Human Immunology Unit, Weatherall Institute of Molecular Medicine, Oxford University, Oxford, UK; ${ }^{2}$ Bioinformatics and Genomics Core Research Groups, Wellcome Trust Centre for Human Genetics, Oxford University, Oxford, UK; ${ }^{3}$ Wellcome Trust Sanger Institute, Hinxton, UK; ${ }^{M}$ MRC Human Immunology Unit, Weatherall Institute of Molecular Medicine and Oxford Sarcoidosis Clinic, Churchill Hospital, Oxford, UK

\section{doi:10.1136/thx.2009.127050b}

Background Sarcoidosis is characterised by granulomatous deposits and hyperactive CD4 T cells. Approximately $60-70 \%$ of patients have disease that resolves spontaneously; the rest follow a chronic course which may result in pulmonary fibrosis. It is unclear why some patients progress and if treatment in this group affects outcome.

Methods We examined expression of 23459 genes from transbronchial biopsies of granulomatous areas of lung samples from histology-proven patients with active but self-limiting ( $\mathrm{S}-\mathrm{L}$ ) pulmonary sarcoidosis compared with those with active, progressive, fibrotic (P-F) disease ( $\mathrm{n}=4$ each). All were never-smokers, not on inhaled or oral medications at the point of sampling, and did not present with Loefgren sydrome. Patients were classified into "S-L" or "P-F" groups on the basis of persistence of symptoms and changes on chext $x$ ray (CXR) over 2 years. "S-L" patients had minor cough, typical nodular change on thoracic high-resolution CT and no change in CXR or symptoms in the ensuing 2 years. "PF" patients presented with some fibrotic areas, an abnormal spirometry or transfer coefficient (KCO), and showed progressive respiratory symptoms over 2 years.

Results Comparison of individual genes identified 133 differentially expressed genes between the two groups. Using Gene-Set Enrichment Analysis, ${ }^{1}$ a powerful, unbiased approach to examine biologically and functionally connected gene sets rather than a single gene transcript, we show a highly significant over-representation of gene sets related to the immune response and cell cycle among genes upregulated in the P-F group (top 20 sets had a false discovery rate (FDR) $\mathrm{q}<0.00002$ ), and a marked enrichment of, and similarity in gene expression profile between hypersensitivity pneumonitis (but not idiopathic pulmonary fibrosis) and P-F sarcoidosis (normalised enrichment score (NES) $=-2.74$, $\mathrm{q}<0.00002)$.

Conclusions The findings suggest that patients with sarcoidosis with P-F disease (1) are more likely to have arrived at this phase via persistent intense immune activity rather than fibrogenic predisposition and (2) have immune mechanisms similar to hypersensitivity pneumonitis, which also signifies the likelihood of disease resolution if these patients are treated early. The findings also demonstrate that microarray expression profiling of transbronchial lung biopsy samples shows a divergent biologically relevant gene profile for the two groups of patients, supporting its potential use as a prognostic classifier for pulmonary sarcoidosis.

\section{Subramaniamn A PNAS 2005.}

\section{S3 INTERSTITIAL LUNG DISEASE IN THE INFLAMMMATORY MYOSITIDES: EXACERBATIONS OF MUSCLE DISEASE ARE NOT PARALLELED BY WORSENING OF PULMONARY FUNCTION}

F Chua, AM Higton, K McNulty, S Grubnic, EJ Edwards, PDW Kiely. St George's Hospital NHS Trust, London, UK

\section{doi:10.1136/thx.2009.127050c}

Background Interstitial lung disease (ILD) associated with idiopathic inflammatory myositis (IIM) is varied in presentation and prevalence. Factors governing its progression remain incompletely understood. We sought to clarify whether muscle and lung function abnormalities might be associated in a heterogeneous cohort of patients with IIM.

Method All patients treated in the Myositis service between 1999 and 2008 with polymyositis (PM), dermatomyositis (DM), overlap syndrome or anti-Jo-1 syndrome were included in this retrospective analysis. Muscle function was assessed using validated isotonic exercise tests and creatine kinase (CK) measurement.

Results 47 patients (mean \pm SD age at diagnosis $49 \pm 17$ years; median 53) were identified, of whom 17 (36\%) were AfroCaribbean, 21 (45\%) Caucasian and $3(6 \%)$ Asian-Indian; 70\% were female. PM was diagnosed in 28/47 (59.6\%) patients, DM in $14 / 47$ $(29.8 \%)$ and overlap syndrome with an underlying rheumatological disorder in 5/47 (10.6\%). ILD was evident in 20/47 (42.5\%) cases: fibrotic non-specific interstitial pneumonia (NSIP) in $14(70 \%)$, usual interstitial pneumonia (UIP) in $3(15 \%)$, organising pneumonia (OP) in 2 and unclassifiable fibrosis in one case. Patients with ILD were more likely to be black $(11 / 20$ vs $6 / 27, p=0.045)$. Five out of 6 patients with Jo-1 autoantibodies developed fibrotic ILD. Pulmonary symptoms preceded myositis in 5/20 (25\%) patients; contemporaneous muscle-lung presentation occurred in another 6 


\section{Abstract S3 Table}

\begin{tabular}{|c|c|c|c|}
\hline & No ILD $(n=27)$ & ILD $(n=20)$ & p Value \\
\hline Female & 19/27 (70\%) & $14 / 20(70 \%)$ & 0.98 \\
\hline Age & $51 \pm 17$ & $45 \pm 17$ & 0.19 \\
\hline \multicolumn{4}{|l|}{ Ethnicity } \\
\hline Black & $6 / 27(22 \%)$ & $11 / 20(55 \%)$ & 0.09 \\
\hline White & $15 / 27(55 \%)$ & $6 / 20(30 \%)$ & \\
\hline Indian & $3 / 27(11 \%)$ & $0 / 20(0 \%)$ & \\
\hline Other & $3 / 27(11 \%)$ & $3 / 20(15 \%)$ & \\
\hline \multicolumn{4}{|l|}{ Myositis diagnosis } \\
\hline PM & $18(67 \%)$ & $10(50 \%)$ & 0.51 \\
\hline DM & $7(26 \%)$ & $7(35 \%)$ & \\
\hline Other & $2(7 \%)$ & $3(15 \%)$ & \\
\hline ANA positive & $16 / 27(59 \%)$ & $14 / 20(70 \%)$ & 0.65 \\
\hline Anti-Jo antibodies & $1 / 27(4 \%)$ & $5 / 20(25 \%)$ & 0.09 \\
\hline CK at presentation & $3063 \pm 5839$ & $3396 \pm 3192$ & 0.52 \\
\hline ESR at presentation & $30 \pm 23$ & $45 \pm 37$ & 0.17 \\
\hline \multicolumn{4}{|l|}{ Survival } \\
\hline 1 year & $27 / 27(100 \%)$ & $20 / 20(100 \%)$ & \\
\hline 3 years & $25 / 25(100 \%)$ & $17 / 18(94 \%)$ & 0.48 \\
\hline 5 years & $13 / 16(81 \%)$ & $15 / 16(94 \%)$ & \\
\hline
\end{tabular}

ANA, antinuclear antibody; CK, creatine kinase; DM, dermatomyositis; ESR, erythrocyte sedimentation rate; ILD, interstitial lung disease; PM, poliomyositis.

(30\%). Baseline restrictive lung function (mean forced expiratory volume in $1 \mathrm{~s}\left(\mathrm{FEV}_{1}\right) /$ forced vital capacity (FVC) ratio 0.81$)$ was evident in $16 / 20$ cases. Mean $\mathrm{DLCO}(\mathrm{c})$ (diffusing capacity) for the group was $50 \pm 17 \%$ predicted. At an average lung function followup of 4.7 years, mean FVC and total lung capacity (TLC) changed minimally $(<10 \%$ from baseline) but DLCO had declined by $\geqslant 10 \%$. Myositis deterioration, characterised by decreased $30 \mathrm{~s} 1 \mathrm{~kg}$ arm lift and chair stand repetitions, and elevated CK, was not was not necessarily accompanied by a deterioration in lung function.

Conclusions Afro-Caribbean patients with inflammatory myositis may have an increased predilection for developing fibrotic ILD. In this study, NSIP was the most common pattern of ILD encountered. Even when it preceded overt myositis, ILD in IIM followed a less aggressive course than in idiopathic disease, with a longitudinal behaviour that did not mirror the exacerbative tendency of the underlying autoimmune myositis.

\section{S4 IDENTIFYING ANXIETY AND DEPRESSION IN INTERSTITIAL LUNG DISEASE: USE OF A SIMPLE OUTPATIENT SCREENING TOOL}

MD Shipley, T Hardy, K Heslop, IA Forrest. Newcastle Hospitals NHS Foundation Trust, Newcastle, UK

\section{doi:10.1136/thx.2009.127050d}

Introduction For many patients with interstitial lung disease, symptom control is the focus of treatment. Psychological symptoms compound the emotional and physical symptoms of respiratory illness. Anxiety and depression are associated with chronic illness but their presence has not been demonstrated in unselected patients with interstitial lung diseases. We have examined the use of an outpatient screening test to identify undiagnosed and untreated anxiety and depression in an interstitial lung disease population.

Methods 44 consecutive patients with interstitial lung diseases attending an outpatient clinic between 1 January 2008 and 31 December 2008 were offered self-screening using the Hospital Anxiety and Depression (HAD) scale. 38 patients completed the assessment. 16 (42.1\%) were male and 22 (57.9\%) were female. Average age was 65.64 years. Average forced vital capacity (FVC) was $77.8 \%$ and average transfer coefficient (KCO) was $89.6 \%$. Two of $38(5.3 \%)$ patients were treated with long-term oxygen therapy. Nine of $38(23.7 \%)$ had a history of ischaemic heart disease. Two of $38(5.3 \%)$ were known to have depression.

Results Diagnosis and incidence of anxiety and depression are shown in fig $1.37 / 38$ (97\%) had symptoms of depression and 35/38 $(92 \%)$ had symptoms of anxiety. Clinically significant levels of depression (10/38 (26.3\%)) and anxiety (15/38 (39.5\%)) were found in the study population. Depression and anxiety were present in males and females and across diagnostic groups. Anxiety was more

\section{Rates of Depression and Anxiety measured by HAD score according to Diagnosis}

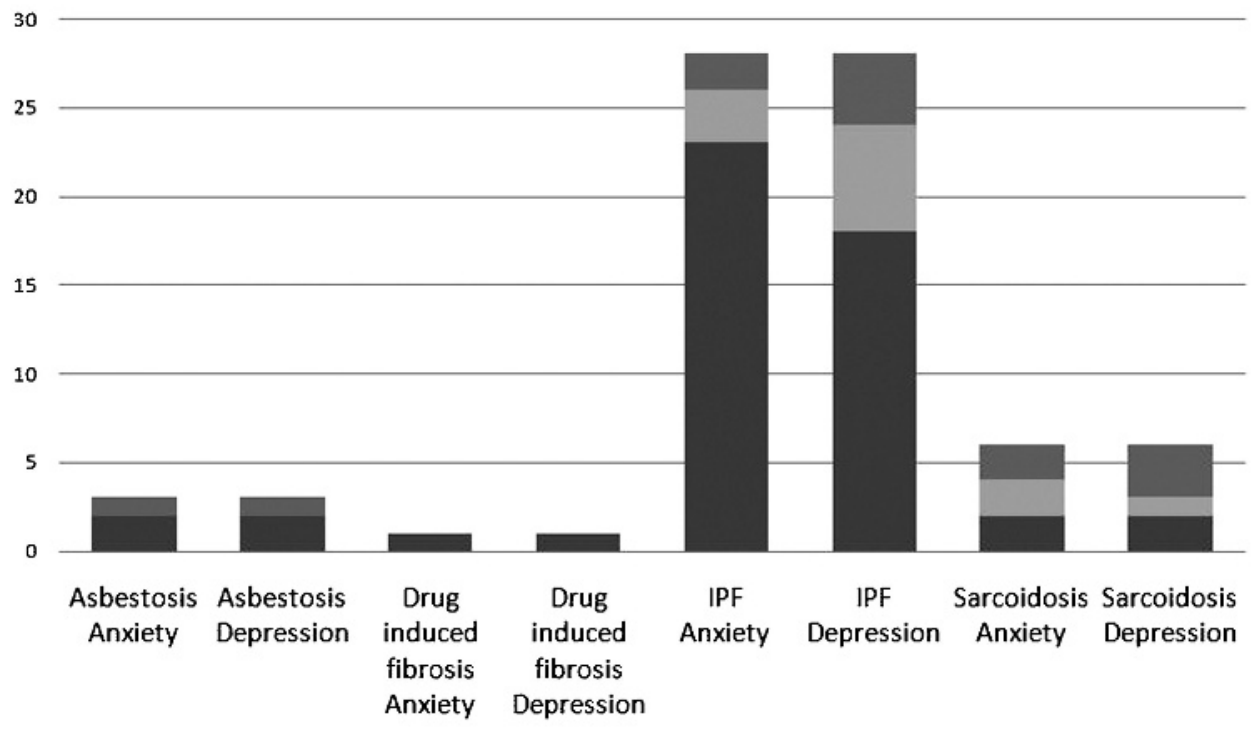

$\square$ 0-7 (No Anxiety or Depression) $=8-10$ (Borderline Anxiety or Depression) $=11-21$ (Anxietyor Depression Present) 
common in patients aged $>65(p=0.048)$. Anxiety appeared more common in females, but this did not reach statistical significance. The presence of depression and anxiety was not related to severity of lung disease as measured by KCO, FVC or oxygen saturations. Conclusions Use of a simple self-administered screening tool can identify high levels of anxiety and depression in outpatients with interstitial lung disease. Anxiety levels are more common in elderly patients. Identifying psychological problems in this patient group may allow development of new therapeutic options so physicians can improve patient's symptoms and quality of life. Psychopharmacology, cognitive behavioural therapy or pulmonary rehabilitation may be possible treatment options and merit further study.

\section{S5 PHYSIOLOGICAL DETERIORATION VS STABILISATION IN MYOSITIS-ASSOCIATED INTERSTITIAL LUNG DISEASE: PHENOTYPIC DIFFERENCES AND INFLUENCE OF IMMUNOSUPPRESSIVE TREATMENT}

AM Higton, PDW Kiely, K McNulty, S Grubnic, EJ Edwards, F Chua. St George's Hospital NHS Trust, London, UK

\section{doi:10.1136/thx.2009.127050e}

Background Interstitial lung disease (ILD) may form a prominent component of idiopathic inflammatory myositis (IIM) and even dominate the clinical course of disease. We observed that certain characteristics were associated with longitudinal worsening of lung disease, including temporal differences that suggest that particular patterns of ILD progression may be expected.

Methods Patients on the St George's Hospital Myositis database with polymyositis (PM), dermatomyositis (DM), overlap syndrome

\section{Abstract S5 Table}

\begin{tabular}{|c|c|c|c|}
\hline & Deteriorators & Stabilisers & \\
\hline & $\mathrm{n}=7$ & $\mathrm{n}=10$ & p Value \\
\hline Age at diagnosis & $39 \pm 15$ & $50 \pm 19$ & 0.18 \\
\hline \multicolumn{4}{|l|}{ Ethnicity } \\
\hline Black & $4(57 \%)$ & $5(50 \%)$ & \\
\hline White & $1(14 \%)$ & $5(50 \%)$ & 0.14 \\
\hline Other & $2(29 \%)$ & 0 & \\
\hline \multicolumn{4}{|l|}{ ILD pattern on HRCT } \\
\hline UIP & $2(29 \%)$ & $4(40 \%)$ & \\
\hline NSIP & $5(71 \%)$ & $4(40 \%)$ & 0.33 \\
\hline $\mathrm{OP}$ & 0 & $2(20 \%)$ & \\
\hline $\begin{array}{l}\text { Presence of lung disease at initial } \\
\text { presentation }\end{array}$ & $5(71 \%)$ & $3(30 \%)$ & 0.15 \\
\hline \multicolumn{4}{|l|}{ Muscle disease } \\
\hline Polymyositis (PM) & $1(14 \%)$ & $8(80 \%)$ & \\
\hline Dermatomyositis (DM) & $5(71 \%)$ & $1(10 \%)$ & 0.008 \\
\hline Other & $1(14 \%)$ & $1(10 \%)$ & \\
\hline ANA positive & $5(71 \%)$ & $6(60 \%)$ & 1.0 \\
\hline Death & $1(14 \%)$ & $4(40 \%)$ & 0.34 \\
\hline \multicolumn{4}{|l|}{$\%$ change in PFTs over first year } \\
\hline FVC & $+7 \pm 21 \%$ & $+17 \pm 30$ & 0.48 \\
\hline DLCO & $-13 \pm 13 \%$ & $+26 \pm 40$ & 0.06 \\
\hline TLC & $-2 \pm 22 \%$ & $+3 \pm 13$ & 0.66 \\
\hline \multicolumn{4}{|c|}{$\begin{array}{l}\% \text { change in PFTs over total duration of } \\
\text { follow-up }\end{array}$} \\
\hline FVC & $-1 \pm 52 \%$ & $+12 \pm 21$ & 0.56 \\
\hline DLCO & $-31 \pm 15 \%$ & $+10 \pm 20$ & 0.002 \\
\hline TLC & $-10 \pm 26 \%$ & $+2 \pm 15$ & 0.37 \\
\hline
\end{tabular}

ANA, antinuclear antibody; DLCO, diffusing capacity; FVC, forced vital capacity; HRCT, highresolution $C T$; ILD, interstitial lung disease; NSIP, non-specific interstitial pneumonia; $\mathrm{OP}$, organising pneumonia; PFT, pulmonary function test; TLC, total lung capacity; UIP, usual interstitial pneumonia. or antisynthetase (Jo-1) syndrome with associated pulmonary abnormalities (as confirmed by CT) were identified. Stable lung disease was accepted as a $\leqslant 10 \%$ decrease in forced vital capacity (FVC) and/or a $\leqslant 15 \%$ decrease in diffusing capacity (DLCO) over the first year of diagnosis.

Results 20 patients with IIM-ILD were identified (mean FVC $70 \pm 18 \%$ predicted, mean DLCO $50 \pm 17 \%$ predicted at diagnosis). Mean duration of myositis follow-up was $10.75 \pm 6.4$ years. 17 of 20 $(85 \%)$ had $\geqslant 2$ measurements of pulmonary function tests (PFTs), with an average total PFT follow-up of 4.7 years. DLCO decline was evident in 7 patients, against a stable/improved pattern in 10 others. Deteriorators tended to have a greater decline in DLCO in their first year of follow-up $(-13 \pm 13 \mathrm{vs}+26 \pm 40$ for stable patients $\mathrm{p}=0.06$ ). Overall, most patients who experienced early PFT deterioration continued to do so thereafter, albeit at a variable rate. For most, an established decline in DLCO at 3 years from diagnosis heralded further loss subsequently. Deteriorators tended to be younger and more commonly reported respiratory symptoms at initial diagnosis. Amongst them, DM was the most common myositis $(5 / 7,71 \%)$, in contrast to $8 / 10(80 \%)$ of stable patients with PM $(p=0.008)$. Afro-Caribbean ethnicity was also more frequent in those with IIM-ILD but did not distinguish deteriorators from stabilisers. Patient numbers were inadequate for ascribing clinical significance to specific ILD patterns.

Conclusions For most patients with myositis, ILD is a relatively benign complication that is satisfactorily controlled by immunosuppressive therapy. In those with progressive lung disease, loss of DLCO appears to be a crucial consequence. These individuals more commonly have DM, are younger and have a shorter duration of lung disease. PFT stabilisation following early deterioration is occasionally apparent, although the long-term significance of this observation remains unclear.

\section{Cellular interactions in the pathogenesis of pulmonary hypertension}

\section{S6 REGULATION OF ENDOTHELIN-1 PRODUCTION BY THE TRANSFORMING GROWTH FACTOR/BONE MORPHOGENETIC PROTEIN PATHWAY IN HUMAN PULMONARY ARTERY SMOOTH MUSCLE CELLS}

${ }^{1}$ PM de Souza, ${ }^{2} N W$ Morrell, ${ }^{2}$ PD Upton, ' JES Park, 'SJ Wort. 'Unit of Critical Care, NHLI, Imperial College, London, UK; ' University of Cambridge School of Clinical Medicine, Addenbrooke's/CUHNHSFT and Papworth Hospitals, Cambridge, UK

\section{doi:10.1136/thx.2009.127050f}

Background Pulmonary artery hypertension is a fatal condition associated with remodelling of pulmonary resistance vessels. There is convincing evidence for the involvement of both the transforming growth factor $\beta$ (TGF $\beta$ )/bone morphogenetic protein (BMP) and endothelin (ET-1) pathways in this remodelling process. However, it is unknown how these two pathways interact.

Aim To investigate the effect of TGF $\beta 1$, BMP2 and BMP4 on ET-1 release from normal human pulmonary artery smooth muscle cells (HPASMCs).

Methods HPASMCs were grown from resected and morphologically normal pulmonary arteries taken from patients with lung cancer at the Royal Brompton Hospital. Cells were treated with TGF $\beta 1$ and/ or BMP2 and BMP4 (0, 1 and $10 \mathrm{ng} / \mathrm{ml})$. Following $24 \mathrm{~h}$ incubation supernatants were collected and ET-1 concentrations determined by ELISA (R\&D, Abingdon, UK). Data were analysed using Student $t$ test.

Results TGF $\beta 1$ dose dependently increased ET-1 release from HPASMCs. TGF $\beta 1(1 \mathrm{ng} / \mathrm{ml})$ significantly increased ET-1 generation by $553 \%$ compared with cells treated with medium alone (fig 1 ; $\mathrm{n}=6))$. BMP2 $(10 \mathrm{ng} / \mathrm{ml})$ and BMP4 (1 and $10 \mathrm{ng} / \mathrm{ml})$ also 